\title{
Prototipo de herramienta tecnológica para el registro, almacenamiento, transmisión y análisis de la información de accidentes de tránsito
}

\section{Prototype of technological tool for the registration, storage, transmission and analysis of traffic accident information}

\author{
Dolly Smith Flórez-Moreno \\ Universidad Santo Tomás \\ Bucaramanga, Colombia \\ dolly.florez@ustabuca.edu.co \\ Carlos Felipe Reyes-Contreras \\ Universidad Santo Tomás \\ Bucaramanga, Colombia \\ carlos.reyes@ustabuca.edu.co
}

\author{
Saul Eduardo Villamizar-Camelo \\ Universidad Santo Tomás \\ Bucaramanga, Colombia \\ saulvillamizar.sv@gmail.com
Sergio Andrés Zabala-Vargas
Universidad Santo Tomás
Bucaramanga, Colombia
Sergio.zabala@ustabuca.edu.co

\begin{abstract}
Resumen- La importancia del correcto registro, almacenamiento y seguimiento de información asociado a accidentes de tránsito es clave para la toma de decisiones en áreas tan diversas como salud pública, políticas viales, seguros, entre otros. La legislación colombiana ha delegado a las entidades responsables del tránsito la implementación de herramientas tecnológicas que faciliten la documentación del Informe Policial de Accidentes de Tránsito. El presente proyecto desarrolla una solución fundamentada en un aplicativo software que permite digitalizar el registro, hasta ahora manual, de la información de los accidentes y su posterior visualización y análisis en los sistemas de información. La investigación demuestra que existen mejoras en los tiempos de registro de la información y en la confiabilidad de los datos. Se requiere profundizar en los procesos de formación de los usuarios en competencias digitales para su uso, así como en la mejora del proceso de levantamiento del croquis del accidente.
\end{abstract}

Palabras clave- Accidentes de tránsito, informe policial de accidente de tránsito, sistemas de información viales.

Abstract- The correct registration, storage and monitoring of information associated with traffic accidents is key to decision making in areas as diverse as public health, viable policies, insurance, among others. Colombian legislation has delegated to the entities responsible for transit the implementation of technological tools that facilitate the documentation of the Traffic Accident Police Report. The present project develops a solution based on software application that allows to digitize the registry, until now manual, of the information of the accidents and its later visualization and analysis in the information systems. The research shows there are improvements in the time of recording information and in the reliability of the data. It is necessary to deepen the processes of training users in digital skills for their use, as well as in improving the process of lifting the sketch of the accident.

Keywords- Traffic accidents, Traffic accident police report, road information systems.

\section{INTRODUCCIÓN}

Según estadísticas de la Organización Internacional de Fabricantes de Vehículos de Motor - OICA, Colombia contaba en 2015 (cifra más recientemente publicada) con cerca de 111 vehículos por cada 1000 habitantes [1]. Esta cifra ubica a CoIombia con una baja densidad vehicular (puesto 94 entre 141 países); sin embargo, esto no redunda en indicadores de accidentalidad bajos. Por ejemplo, en 2017 el Instituto Nacional de Medicina Legal y Ciencias Forenses registró 6.754 personas fallecidas y 40.115 con lesiones no fatales, para un total de 46.869 casos atendidos [2]. Esto sin considerar todos aquellos casos en los cuales ocurren accidentes de tránsito y no se reportan a las autoridades. 
Las situaciones descritas en el párrafo anterior han sido consideradas por el Gobierno nacional, el cual ha generado el Código Nacional de Tránsito Terrestre, reglamentado con la Ley 769 de 2002 y posteriormente reformada por la Ley 1383 de 2010. Dicha Ley ha establecido el Registro Único Nacional de Tránsito (RUNT) y el Registro Nacional de Accidentes de Tránsito (RNAT). En esta legislación se comprende el accidente de tránsito como: "Evento, generalmente involuntario, generado al menos por un vehículo en movimiento, que causa daños a personas y bienes involucrados en él..."; y se declara la importancia de realizar el registro, monitoreo, seguimiento y prevención de estas situaciones, las cuales se convierten en afectaciones a la salud pública [3], [4].

Es en este sentido que la Resolución del Ministerio de Transporte 11268 de 2012 reglamenta el Informe Policial de Accidentes de Tránsito (IPAT), como estrategia para unificar el registro de los accidentes de tránsito y facilitar el almacenamiento en los sistemas de información nacional. En el Manual de diligenciamiento del IPAT propuesto en la citada Resolución se presenta la importancia de contar con un "procedimiento técnico veraz y efectivo", que pueda generar: 1. Bases jurídicas sólidas a las autoridades para poder resolver y dirimir el accidente. 2. Facilitar el diligenciamiento de la información en el sistema RNAT. 3. Realizar acciones ágiles y precisas para atender a los afectados y poder salvaguardar la vida de estos. 4. Tomar las medidas de seguridad vial necesarias. 5 . Utilizar los medios de comunicación disponibles para generar evacuación rápida de los afectados. 6. Evitar entrar en contacto con personas fallecidas, sus documentos o pertenencias. 7. Identificar a los conductores involucrados en el hecho y sus documentos de identidad. 8. Colaborar con las partes involucradas sin tomar partido ni generar juicio de valor, entre otros [5].

Ahora bien, para lograr este propósito, el Ministerio de Transporte de Colombia habilita a las autoridades de tránsito para "implementar nuevas tecnologías que permitan la captura, diligenciamiento, almacenamiento y lectura de la información contenida en el Informe Policial de Accidentes de Tránsito (IPAT)" [5], [6]; siendo esta una posibilidad para que investigaciones como las presentadas en este artículo sean posibles, no solo a nivel de desarrollo y adaptación de herra- mientas tecnológicas, sino también en aportes al mejoramiento de los procedimientos.

En este orden de ideas, se realizó una indagación preliminar en fuente primaria, recurriendo al equipo de levantamiento de accidentes de la Dirección de Tránsito y Transporte de Bucaramanga. Se recurrió a un grupo focal donde se discutió sobre el procedimiento actual y las oportunidades de mejora que, los directamente implicados, han detectado. Entre las más importantes se encuentran: 1) Aunque existe una Ley reglamentaria para el registro de accidentes a través del sistema de información IPAT, el procedimiento es manual, generando reescrituras que se traducen en lentitud de registro y errores en los datos que finalmente se almacenan en los sistemas de información. 2) No se cuenta con una metodología validada (en muchos casos meramente empírica) para que los agentes de tránsito realicen un levantamiento adecuado de la información de un accidente, registrando consideraciones importantes como estado de la vía, clima, situaciones exógenas, entre otros y 3) La trazabilidad y generación de balances del estado real de la accidentalidad en Bucaramanga es bastante reducida; siendo esto una limitante para la generación de campañas adecuadas de prevención. El personal encuestado reconoce la existencia de orientaciones internacionales como las ofrecidas por la Organización Mundial de la Salud sobre vigilancia de lesiones y violencia [7]; sin embargo, no cuenta con elementos claros ni documentados para aplicar realmente estrategias fiables al respecto.

Los elementos anteriores muestran que existen retos procedimentales, metodológicos y tecnológicos; lo que motivó a hacer una revisión de antecedentes en el tema. Se recurrió a un ejercicio de revisión de estado de arte en las bases de datos Scopus e IEEE, con una ventana temporal de 10 años y un criterio de inclusión de artículos completos y publicaciones en conferencias. Los resultados de esta revisión se sintetizan a continuación:

La primera categoría detectada se asocia a las investigaciones que relacionan los accidentes de tránsito y, los problemas de tráfico vehicular que afectan la salud y calidad de vida de las comunidades. En [8] se presenta una experiencia desarrollada para el Centro de Estudios del Transporte 
de la Universidad de Virginia, donde se argumenta que los problemas de tráfico son un problema de alto costo, tanto del punto de vista del usuario como del responsable de la administración del tráfico. En dicha investigación se valoran tres tipos de modelos para la predicción del clearance time (tiempo de levantamiento, asociado en el caso de publicación a un accidente de tránsito): un modelo estocástico, un modelo de regresión no-paramétrico y un modelo de clasificación de árbol. La investigación permite observar que las dos primeras alternativas no dan resultados pertinentes con información de prueba, sin embargo, la última metodología permite obtener resultados interesantes para la predicción propuesta. Una segunda experiencia es la diseñada por [9], en la que presenta el uso de la shockwave theory (Estrategia de análisis que permite realizar estimaciones del flujo, en este caso de vehículos, en un entorno real; considerando situaciones de frontera y comportamientos cambiantes a través del tiempo y el espacio). Esta estrategia hace estimar los tiempos de disipación de los efectos de un accidente de tránsito, con una eficiencia estadística superior al 95\%. En la valoración de la relación entre los accidentes de tránsito y la operación del tráfico en áreas urbanas, es posible citar el trabajo mostrado en [10], donde se resalta cómo el tiempo de recuperación del tráfico luego de un accidente puede ser estudiado a través del análisis de vehículos que arriban vs. vehículos que salen del punto de limitación de flujo. Estos autores proponen modelos matemáticos, basados en optimización, que permiten estimar la acumulación de vehículos; sin embargo, no se presentan validaciones en condiciones reales. En [11] se ilustra una investigación utilizando minería de datos, recurriendo a las herramientas H2O y WEKA, sobre una base de datos con más de 140.000 ejemplos de accidentes; permitiendo estimar comportamiento del conductor en la carretera y el pronóstico de lugares con alta accidentalidad. Esta propuesta aborda temáticas de interés para el presente proyecto como los Sistemas de Transporte Inteligentes (ITS) y las redes Ad-hoc vehiculares (VANET). Este último trabajo enseña elementos claves, como: 1. Cerca de un 65\% de los accidentes ocurren en zonas residenciales con límites de velocidad de 30 $\mathrm{km} / \mathrm{h}$; es decir que la gran cantidad de accidentes no suceden en las autopistas de alta velocidad. 2. Un $65.8 \%$ de los accidentes ocurren en zonas urbanas, pero cerca del 70\% son "suaves". Mientras que en el caso rural hay un porcentaje mayor de accidentes "graves" y 3. La mayor cantidad de accidentes ocurre, contrario a lo que se puede pensar a través del sentido común, en estados climáticos favorables (buen tiempo sin lluvia o nieve). Finalmente, en [12] se presenta un análisis matemático que modela el tiempo de duración de los problemas de tráfico asociado a un accidente de tránsito. El modelo considera aspectos como el largo de la vía, el ancho de esta, la longitud del accidente, el flujo del tráfico entre otros aspectos.

Una segunda categoría se asocia con el uso de redes VANET y otras aplicaciones de comunicación para el monitoreo y supervisión de accidentes, así como el control de tráfico. En [13] se presenta una propuesta que integra el uso de sensores y radio bases en las intersecciones, con el fin de poder dar soporte a cada automotor de la proximidad de otro(s) en el cruce al que se acerca. La propuesta aborda el uso del Algoritmo Fisher para el registro de movimiento de los objetos dinámicos (en este caso vehículos) y poder determinar el punto de encuentro. La propuesta fue validada en condiciones de laboratorio. En [14] se presenta una experiencia de implementación de redes VANET para el intercambio de información entre usuarios que se encuentran en vehículos en movimiento, así como la comunicación con proveedores de servicio y sistemas de emergencias. Una revisión más profundiza sobre el tema de las VANET y sus categorías (redes vehículo-vehículo, redes vehículo-infraestructura e híbridas) se muestra en el artículo de revisión presentado en [15]. Entre las principales conclusiones de los autores relacionados se encuentra la importancia de seguir fortaleciendo el desarrollo de protocolos específicos para este tipo de red y aprovechar las capacidades de Gateway móviles antes que desplegar una nueva infraestructura. Otras experiencias importantes se asocian a algoritmos de seguridad de la información y su importancia en las redes VANET [16], sistemas de respuesta a emergencias a través de sensores instalados en los vehículos [17], sistemas de detección de velocidad y toma de decisiones con apoyo en servidores en la nube [18] y en la integración de los conceptos de Internet of Things (IoT) y Big Data en las redes de soporte de gestión de tráfico y reducción de accidentes [19]-[21]. 
El uso de video e imágenes para registro y trazabilidad de accidentes de tránsito es una tercera categoría que surge de la revisión. En [22] se presenta una alternativa a la predicción de accidentes en intersecciones de vías, utilizando el registro y procesamiento de video. Para tal fin centran sus esfuerzos en el desarrollo de un algoritmo de detección de accidente como base para un sistema de grabación y reporte de accidentes. Otras estrategias utilizadas para el procesamiento de la información de video son: 1. Hidden Markov Model (HMM) y el método de Dynamic Hierarchical Clustering (DHC) para hacer el reconocimiento de la trayectoria de los objetos (en este caso vehículos, personas, materiales, entre otros) [23]. 2. Campo de Movimiento Denso, PCA direccional y Máquinas de Soporte Vectorial (SVM), con el objeto de discriminar entre una anomalía y un evento normal [24]. 3. Matrices de bajo rango fundamentadas en el hecho de que los accidentes ocurren en una parte muy pequeña de la imagen registrada. La extracción de esta imagen es comparada con escenas de tráfico normal identificando las transiciones bruscas (cambios considerables) en el perfil de esta [25]. Estrategias que integran las redes VANET, el procesamiento de video, el empaquetamiento de paquetes de video; orientado a la mejora de la calidad del servicio son presentados en [26]-[29].

La última categoría de los trabajos revisados se asocia al uso de drones o vehículos aéreos no tripulados (UAV) para realizar la captura de imágenes, y facilitar el procesamiento de información; orientado al mejoramiento de las situaciones en las vías. En la experiencia presentada en [30] se revisa la integración de los sistemas de visión artificial (AVS) en UAV; permitiendo facilitar el control del tráfico en las vías y el análisis automático de accidentes. Para tal fin, se hace el registro de las imágenes, el escalamiento de estas, la generación de la región de interés (ROI), la segmentación de esta, el filtrado y generación de descriptores, para finalmente lograr el reconocimiento de vehículos y sus velocidades. En [31] se presenta una alternativa de procesamiento de video basado en algoritmos de seguimiento (tracking), específicamente el algoritmo Mean Shift, con el que se logran rápidos tiempos de convergencia y altas tasas de detección. En [32] se introduce una solución para el monitoreo de tráfico utilizando siste- mas de radar e imágenes obtenidas de sistemas no tripulados. El eje central de esta investigación es la posibilidad de obtener imágenes de amplias zonas de terreno utilizando los UAV, e integrando con radares de apertura sintética (SAR) y sistemas de indicación de movimiento de objetos en terreno (GMTI). Finalmente, es posible encontrar una revisión del estado de arte del uso de UAV en el monitoreo de tráfico en [33].

En el marco de este amplio panorama de estrategias que pueden aportar al monitoreo y trazabilidad de los accidentes de tránsito, y orientado por las necesidades específicas presentadas por la población objetivo; esta investigación propone como objetivo central: determinar estrategias y lineamientos tecnológicos y metodológicos, que permitan el mejoramiento del proceso de registro, almacenamiento, transmisión y análisis de los accidentes de tránsito; en coherencia con lo reglamentado para el Informe Policial de Accidente de Tránsito - IPAT. Para tal fin, se llevó a cabo una metodología de investigación cualitativa, donde se busca observar cómo la incorporación de herramientas tecnológicas mejoran en el desempeño de la implementación práctica del IPAT, así como el mejoramiento de la confiabilidad de los datos registrados en el sistema RNAT.

\section{METOdOLOGÍA}

La experiencia presentada en este artículo se desarrolló en un conjunto de cinco fases, las cuales son detalladas a continuación: 1 . Construcción de un marco de referencia sobre los antecedentes legales y la descripción general del Informe Policial de Accidentes de Tránsito. 2. Desarrollo del estado de arte de la temática tanto en aspectos tecnológicos como de procedimiento. 3. Levantamiento de requerimientos tecnológicos específicos para registrar la información del accidente en campo, transmisión, el registro en los sistemas de información y el análisis de los hechos. 4. Diseño de la arquitectura de la solución tecnológica determinando requerimientos de infraestructura, funcionales y no funcionales de aplicativo software de apoyo. Y 5. Desarrollo y validación de un prototipo piloto que facilite la implementación del IPAT. La población objetivo de la investigación fue la Dirección de Tránsito y Transporte de Bucaramanga, Colombia, quienes actuaron como expertos meto- 
dológicos del proceso de registro del IPAT y como beneficiarios de solución tecnológica.

A continuación se relaciona con detalle cada una de las fases:

\subsection{Construcción de un marco de referencia sobre los antecedentes legales y la descrip- ción general del Informe Policial de Acciden- tes de Tránsito}

Es posible remontar los antecedentes del Informe Policial de Accidentes de Tránsito - IPAT al Decreto 1344 de 1970 del Ministerio de Justicia, que estableció el Código Nacional de Tránsito Terrestre, así como a la Resolución 5593 de 1993 del Ministerio de Transporte, que adopta el primer manual para el diligenciamiento del Formulario de Informe de Accidente. Es la Resolución 8749 de 2011 que delega a las autoridades de tránsito y transporte de cada municipio del país para expedir el Formulario Informe de Accidente. Es importante resaltar que el IPAT se convierte, según el artículo 8 de la Ley 769 de 2002, en un insumo del Registro Nacional de Accidente de Tránsito (RNAT) que a su vez es parte importante de la información contenida en el Registro Único Nacional de Tránsito (RUNT). Finalmente, como ya fue citado, es la Resolución 111268 de 2012 la que deroga a la Resolución 1814 del 2005, que establece la adopción del Informe Policial de Accidentes de Tránsito (IPAT) y del Manual de Diligenciamiento; y será el principal referente legal del presente documento.

Ahora bien, haciendo referencia al IPAT, el mismo cuenta con tres grandes secciones: En la primera sección (hoja) se consigna la información general del accidente, como es la descripción del lugar, circunstancias, características de la vía, identificación de conductores, vehículos, entre otros. En la segunda sección (hoja) se registra la información del segundo vehículo involucrado, víctimas, hipótesis, testigos y observaciones, y en la tercera sección se presenta el croquis para la identificación de aspectos relevantes del accidente. Existen también dos anexos que dan soporte al Informe completo (Anexo 1. Conductores, vehículos y propietarios, y Anexo 2. Víctimas). Un extracto de la primera página del IPAT es presentado en la Fig. 1. [6].

Una consideración particular se debe tener en cuenta con el denominado croquis, principalmen- te por la importancia que este tiene al momento de comprender la situación que enmarcó el accidente y como base en los procedimientos jurídicos consecuencia del siniestro. La baja calidad en los bosquejos del croquis genera problemas legales, principalmente por la ilegibilidad de este, la posibilidad de no contar con detalles técnicos completos y generar dudas razonables. Esto conlleva, como cita [34] a medidas erradas, dificultad en la investigación y reconstrucción del accidente y la falta de idoneidad a la hora de sustentar un juicio.

Fig. 1. EXTRACTO DEL IPAT- SECCIÓN 1

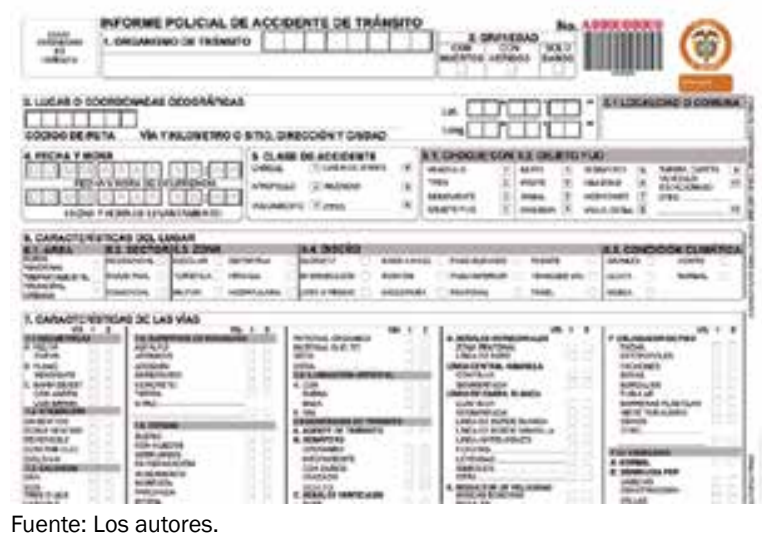

\subsection{Desarrollo del estado de arte de la temá- tica tanto en aspectos tecnológico como de procedimiento}

Los resultados más importantes de esta fase del proyecto fueron presentados en la sección de introducción del presente artículo. Se resaltan la revisiones de estados de la cuestión sobre la temática de incorporación de TIC en el seguimiento de accidentes y su relación con las dinámicas de tráficos que fueron encontrados en [15], [33], [35].

\subsection{Levantamiento de requerimientos espe- cíficos para el sistema de apoyo al registro del IPAT}

En esta fase se verificó los requerimientos tecnológicos funcionales y no funcionales para el desarrollo de una solución tecnológica que dé soporte al registro de información del IPAT. Esto se llevó a cabo con la implementación de entrevistas semiestructuradas a parte del equipo de levantamiento de accidentes y de los responsables del registro de la información en los sistemas de información nacional de la DTTB. Posteriormente se 
hizo una reunión plenaria de discusión y concertación de requerimientos. En el siguiente listado se presentan los requerimientos funcionales y no funcionales:

- La solución tecnológica debe permitir el registro de la información, por parte de los agentes de tránsito en campo, de todos los campos mostrados por el IPAT. Para tal fin es necesario que el aplicativo se pueda implementar en un hardware específico de fácil desplazamiento para el usuario, como puede ser un móvil, tablet $u$ otro dispositivo terminal.

- El aplicativo software, base de la solución tecnológica, debe operar en un esquema cliente-servidor; recurriendo a estrategias de comunicación a la red de datos desde el dispositivo terminal al servidor central.

- Se debe garantizar que el aplicativo cuente con al menos tres roles: a. Rol del agente de tránsito, que puede registrar un nuevo caso (accidente), ingresar y modificar información en sitio, realizar el levantamiento del croquis y realizar la entrega del informe a los afectados. Luego de remitir la información a la base de datos central, no puede realizar modificaciones o cambios. El usuario que cuente con este rol solo puede ver los casos que él ha registrado. b. Rol de líder de proceso: el cual se le asigna al directivo responsable del proceso de levantamiento de accidentes de tránsito y de ser el canal formal ante los sistemas de información nacional. Tiene la posibilidad de observar todos los casos y generar estadísticos para la toma de decisiones, y c. Rol de administrador de plataforma: Es un rol de gestión administrativa TI. Está pensado para poder administrar la base de datos y actualizar versiones del aplicativo.

- El aplicativo debe permitir, al finalizar el registro de cada caso, generar la impresión del informe para firma por parte de los involucrados. Se debe contar con la posibilidad de digitalizar el documento firmado y subirlo a plataforma.

- El aplicativo debe contar con la posibilidad de configurar fuentes de letras (tamaño y estilo), así como opciones de autocompletar; considerando que la población principal de usuarios cuenta con edades superiores a los 50 años (>55\% de los agentes de tránsito en 2016).
- Debe permitirse el almacenamiento de datos en un servidor que garantice seguridad y agilidad de acceso. Debe contar con la posibilidad de almacenamiento local para situaciones en las que no se cuenta con acceso a Internet y que permita su posterior envío y sincronización.

- Se debe garantizar una posible concurrencia de mínimo 50 usuarios en la misma ventana de tiempo; considerando que en la DTTB existe un equipo de aproximadamente 20 agentes especializados en el registro de accidentes y se propone considerar un $100 \%$ adicional de concurrencia.

- El aplicativo debe proponer estrategia(s) para la elaboración y almacenamiento del croquis.

- Contar con un panel de usuario en la web que permita visualizar los datos de cada paso, para poder generar informes sobre accidentalidad; permitiendo (posteriormente) la integración con sistemas de información nacional.

- Se propone por los interesados del proyecto la posibilidad de revisar estrategias para facilitar las mediciones de distancias (o corroborarlas si se hacen manualmente) entre los diferentes elementos en la escena del accidente (personas, vehículos, puntos de referencia, entre otros).

\subsection{Diseño de la arquitectura de la solución tecnológica determinando requerimientos de infraestructura, funcionales y no funcionales de aplicativo software de apoyo}

La solución cuenta, desde su arquitectura de operación, con dos puntos principales: a. Dispositivo de registro, el cual permita a cada usuario avalado para el registro del accidente ingresar la información completa por medio digital para ser almacenada y transmitida a puntos de apoyo. En este punto de registro se instala el aplicativo software con el IPAT completo, incluida la estrategia de registro del croquis. b. Servidor en la nube donde se aloja la base de datos para registro de la información completa de cada accidente, así como un servicio web que permite a los interesados, debidamente aprobados, visualizar, procesar, descargar y analizar la información registrada. En la Fig. 2 se presenta un esquema básico de dicha solución. 
Fig. 2. ESQUEMA BÁSICO DE LA ARQUITECTURA DE LA SOLUCIÓN

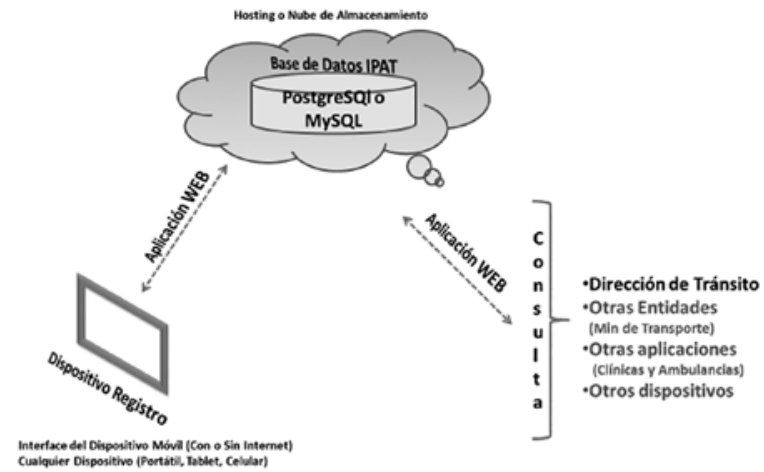

Fuente: Los autores.

\subsection{Desarrollo de un prototipo piloto que fa-} cilite la implementación del IPAT

En esta sección se presentan dos soluciones que se desarrollaron en el marco del proyecto que da origen a este artículo. El primero, denominado IPAT_SOFT, se centra en el proceso de registro del documento completo del IPAT, levantamiento del croquis de forma digital, transmisión de información a base de datos y visualización de resultados. En un segundo aparte, se presenta el prototipo denominado CROQUIS_UAV, el cual se puede integrar al IPAT_SOFT y facilita el registro de evidencias y métricas para el croquis.

\subsubsection{Detalle de la solución IPAT_SOFT para registro del IPAT}

El prototipo desarrollado en el presente proyecto se centra en el aplicativo software desarrollado. Este ha sido desarrollado en plataforma Unity, orientada para el desarrollo de juegos 2D y 3D, principalmente. Se cuenta con una solución multiplataforma (Android, Windows y IOS) y es instalable en cada una de las tablet, móviles o dispositivos terminales seleccionados. El nombre comercial propuesto para el aplicativo es IPAT_SOFT. La base de datos fue desarrollada en MySQL e instalada en servidor en la nube para pruebas de validación.

El diagrama de entidad-relación de la base de datos asocia la información completa de cada uno de los accidentes en una entidad denominada Policia_transito. En esta, es la cédula de ciudadanía del agente de tránsito la llave principal del diseño. También se relaciona esta in- formación con los datos personales del agente, así como con una contraseña personal definida para este por el administrador de la plataforma. Se cuenta también con una entidad denominada vehículo que cuenta con los detalles de cada uno de los involucrados en el accidente. Otra entidad de la base de datos se asocia al detalle de la zona donde ocurre el incidente, así como información relacionada del mismo; la cual se denomina lugar_impacto. Las entidades testigos y víctimas también se relaciona en la base de datos y permite tener detalle de estos campos vinculados al IPAT.

Desde el punto de vista del desarrollo, el proyecto se centra en la creación de assets asociados al entorno Unity, entendidos estos como "una representación de cualquier ítem que puede ser utilizado en su juego o proyecto. Un asset podría venir de un archivo creado afuera de Unity, tal como un modelo 3D, un archivo de audio, una imagen, o cualquiera de los otros tipos de archivos que Unity soporta" [36]. Particularmente el aplicativo fue basado en un conjunto de escenas de alto nivel, interrelacionadas con las guías, los plugins y los assets prefabricados para la integración de la solución completa. En cuanto a las escenas se cuenta con: 1 . Acceso al aplicativo y validación; 2. Formulario para registro de campos; 3 . Croquis digital y 4 . Información general del usuario. La totalidad de los scripts fueron desarrollados en C\#. En la Fig. 3 se presentan imágenes del aplicativo, donde se resaltan los campos para el registro de la totalidad de componentes del IPAT.

Como se citó en el párrafo anterior, uno de los elementos desarrollados es el registro del croquis digital orientado a minimizar problemas de registro, baja calidad gráfica, entre otros problemas que cita [34]. En primera instancia se encuentra la parametrización de elementos como escala, información de la vía (peralte, radio, pendiente), la tabla de medidas y la longitud de las huellas. También la herramienta permite ubicar elementos propios de la escena como personas, vehículos, árboles, entre otros. Se ha incorporado un plugin de Googlemaps con el cual se puede generar, recurriendo al GPS del móvil utilizado, un mapa que facilite el posicionamiento del accidente y el registro del croquis. Un ejemplo de esto es presentado en la Fig. 4. 
Fig. 3. IMÁGENES DEL APLICATIVO SOFTWARE IPAT_SOFT (EJEMPLOS PÁGINA 1 Y 4 DEL IPAT)

\section{Informe policial de accidente de tránsito}

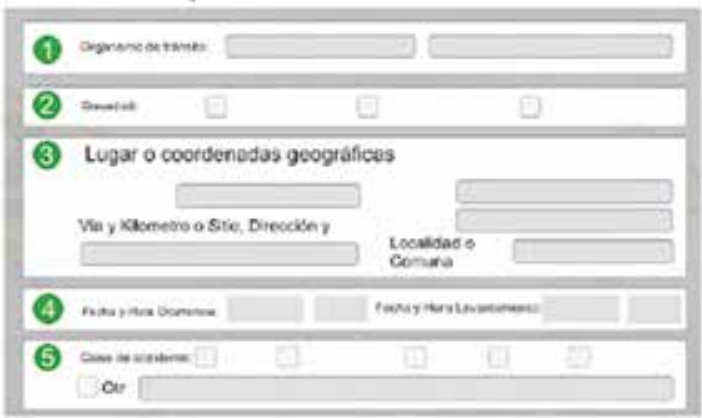

Caracteristicas de las vias

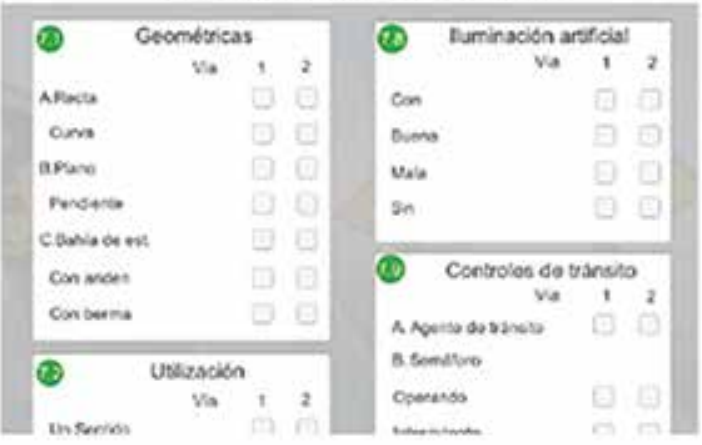

Fuente: Los autores.

Fig. 4. EJEMPLO DE LA TRAZA DEL CROQUIS RECURRIENDO AL PLUGIN DE GOOGLEMAPS

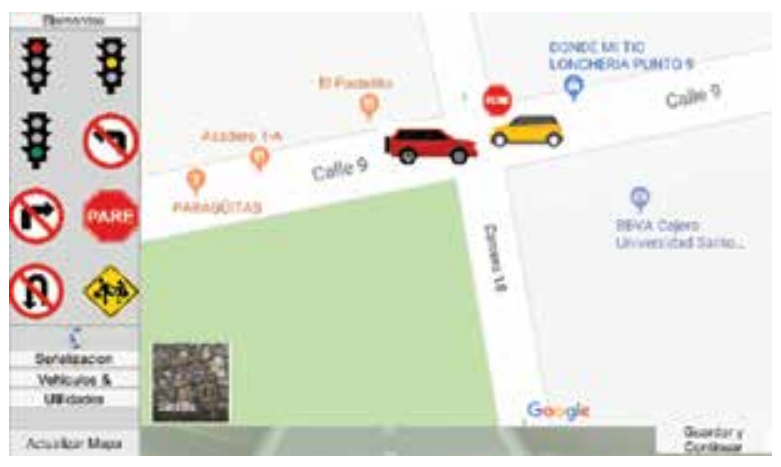

Fuente: Los autores.

\subsubsection{Detalle de la solución CROQUIS_UAV}

Según las experiencias de investigación presentadas en [30], [32], [33], [35], es posible utilizar sistemas UAV (drones aéreos) para facilitar el monitoreo de accidentes de tránsito y poder realizar automatización de procesos, reducción de tiempos y optimización de tráfico. En esta iniciativa se muestra el resultado denominado CROQUIS_UAV, el cual recurre al registro de imágenes aéreas de la zona del accidente (vista superior) para automatizar el registro de distancias entre los objetos.

Se llevó a cabo el registro fotográfico de la zona del accidente a partir de un Parrot Ar. Drone 2.0. Este cuenta con una cámara principal con resolución de 720p (no utilizada en el experimento) y una inferior de $480 p$ con la que se realizaron los registros[37]. Las tomas se hicieron desde una vista superior que permita observar la zona del accidente y sus alrededores, para luego proyectar las distancias (medidas) entre puntos de interés (vehículo-víctima, vehículo-vehículo, víctima-punto de referencia, entre otros). La solución también integra el mando del UAV, por lo cual se puede controlar desde la solución propuesta, realizar el registro de la fotografía y luego realizar el análisis según la escala que le indique el usuario.

CROQUIS_UAV fue desarrollado en Android Studio V2.3, recurriendo a librerías de procesamiento de imágenes, cálculos matemáticos, graficación en pantalla, entre otros. Se contó con adaptación de librerías del software OPENCV para la extracción de características. Básicamente la herramienta toma puntos de referencia y distancias indicadas por el usuario, y calcula según los puntos de interés, las distancias en centímetros/metros de forma automática.

\subsection{Pruebas de validación del funcionamien- to de los aplicativos software desarrollados}

Se han llevado a cabo tres pruebas de validación:

- Prueba 1. Usabilidad (caracterización cualitativa): Se instaló el aplicativo IPAT_SOFT en tres dispositivos (2 tabletas Samsung Galaxy Tab con pantalla de 10.1 pulgadas y un móvil Samsung Note 4 de 5.7 pulgadas). Se llevó a cabo la instalación de la base de datos en el hosting: ipatsoft.com, configurando que todos los terminales orienten la transmisión de datos a dicho repositorio de información. Se contó con el apoyo de tres usuarios (tester) en el levantamiento de dos experimentos para registro de IPAT. Se hizo la capacitación del formulario y la totalidad del registro. Se llevaron a cabo las siguientes fases: 1 . Se preparó un guion (simulación) de un accidente de tránsito con campos predefinidos. Para tal fin 
se toma como ejemplo una prueba con datos sintéticos (Datos 1) construidos con las recomendaciones de un agente de tránsito retirado; 2. Se registró la totalidad del caso en los tres dispositivos, tomando cada uno un agente de tránsito diferente; 3. Se hizo una aproximación inicial al bosquejo de un croquis tomado como referencia; los tres utilizando el canvas (espacio de trabajo) en blanco; 4. Se verificó la información cargada a la base de datos de la plataforma web, realizando el chequeo de la integralidad de la información; 5. Se comparó la información almacenada offline en el dispositivo de registro vs. información almacenada en servidor online; 6 . Se hizo el registro de un segundo accidente de tránsito (Datos 2) intercambiando el tipo de dispositivo entre los usuarios de prueba (móvil y tablet), y 7 . Se aplicó observación cualitativa del comportamiento de la herramienta y la opinión de los tester.

- Prueba 2. Comparación tiempos de diligenciamiento: Se llevó a cabo la comparación entre los tiempos de diligenciamiento del IPAT por parte de 10 usuarios. Debido a que en la población objetivo existe un rango de edad amplio, se hace la selección de usuarios en un rango de edades que oscilan entre 27 a 57 años, con una media de 41 años y una desviación estándar de 10.61. El detalle de las edades es mostrado en la Tabla I. Cabe resaltar que en esta prueba los usuarios seleccionados no contaban con experiencia en el diligenciamiento previo del IPAT, y todos recibieron la misma capacitación basados en la Resolución 11268 de 2012 [6]. Se hizo una prueba cronometrada que compara el tiempo, en minutos del registro de los datos manualmente (hoja física) versus el tiempo que se toma el registro con el aplicativo software. Se comparó entre datos y el cálculo de errores.

- Prueba 3. Calculo error levantamiento croquis con aplicativo: CROQUIS_UAV: Para esta prueba se realizó la simulación de un accidente en un entorno controlado (parqueadero). En este caso se emuló el choque de dos vehículos y se realizó el registro fotográfico, recurriendo al dron, a distancias de a) 5 metros, b) 10 metros, c) 15 metros y d) 20 metros; seleccionando tres distancias de prueba en diferentes puntos de la imagen.
Los resultados de cada una de estas pruebas son presentados en la siguiente sección.

TABLA I

RELACIÓN DE EDADES DE LOS USUARIOS DE PRUEBA

\begin{tabular}{|c|c|}
\hline Id Usuario & Edad [años] \\
\hline Usuario_1 & 27 \\
\hline Usuario_2 & 30 \\
\hline Usuario_3 & 33 \\
\hline Usuario_4 & 34 \\
\hline Usuario_5 & 42 \\
\hline Usuario_6 & 47 \\
\hline Usuario_7 & 49 \\
\hline Usuario_8 & 51 \\
\hline Usuario_9 & 53 \\
\hline Usuario_10 & 57 \\
\hline
\end{tabular}

Fuente: Los autores.

\section{RESULTADOS}

\subsection{Resultados prueba 1}

La prueba realizada arroja como resultados cualitativos los siguientes:

- El registro de información es más ágil cuando se utiliza la tablet, que cuando se lleva a cabo con un dispositivo móvil. Se sustenta principalmente la importancia y comodidad de una pantalla de mayor tamaño para facilitar la lectura y el registro de la información.

- Se compararon los datos almacenados en el dispositivo móvil en cada uno de los dos experimentos (Datos 1 y Datos 2) encontrando $100 \%$ de coincidencias con la información registrada en el servidor web. Esto es coherente con el hecho de que la cantidad de datos por registrar es muy baja comparada con el ancho de banda del canal propuesto (conexión de datos $3 G$ o superior en la zona de registro).

- Los usuarios de la prueba piloto recomiendan utilizar la herramienta de reconocimiento de voz integrada en la plataforma Android para el registro en los campos con mayor cantidad de información.

- Se solicita la revisión del tamaño de las fuentes de la primera versión, y que algunos usuarios indicaron que la misma era muy pequeña. También se hizo la distribución del formato en 
más hojas, disminuyendo la cantidad de campos para diligenciar por pantallazo.

- Se observa dificultad en la realización del croquis, sobre todo, asociado a la comprensión en el uso de la plataforma, tanto en su gestión como en la operatividad de esta. Se verifica que el uso de lápiz óptico facilita la creación del croquis, así como el mejoramiento en la capacitación del usuario.

\section{2 Resultados prueba 2}

En la Tabla II se presenta comparativa de los datos registrados de tipo manual (hoja física) denominados TRM y los registrados de forma automática con el software IPAT_SOFT. Es importante citar que la verificación de los tiempos de registro es fundamental, considerando que además de asegurar la zona y garantizar la correcta gestión de los equipos de emergencia en un accidente (en caso de requerirse), el agente debe resolver el registro lo antes posible.

TABLA II

TIEMPO DE REGISTRO MANUAL (TRM) Y TIEMPO DE REGISTRO AUTOMÁTICO (TRA) VS. ID_USUARIO

\begin{tabular}{|c|c|c|}
\hline Id Usuario & TRM [min] & TRA [min] \\
\hline Usuario_1 & 16,8 & 14,1 \\
\hline Usuario_2 & 14,1 & 11,6 \\
\hline Usuario_3 & 18 & 16,5 \\
\hline Usuario_4 & 16,3 & 13 \\
\hline Usuario_5 & 17 & 15,3 \\
\hline Usuario_6 & 18 & 16,5 \\
\hline Usuario_7 & 18,3 & 17,4 \\
\hline Usuario_8 & 20,1 & 19,7 \\
\hline Usuario_9 & 19,3 & 22,1 \\
\hline Usuario_10 & 19,8 & 23,8 \\
\hline
\end{tabular}

Fuente: Los autores.
Los datos presentan una varianza para TRM de 2,94 y para TRA DE 13,6; siendo 4,6 veces más grande la segunda. Esto, a juicio de los autores, indica que la diferencia de edad está penalizando la agilidad con la que el usuario interactúa con el equipo de cómputo y el aplicativo software. Este aspecto es crítico al momento de implementar la herramienta, ya que se observan casos donde el tiempo de registro automático supera, hasta en un $20,2 \%$, el tiempo de registro manual. Se observa la edad como un factor significativo en los tiempos de registro, tanto de forma manual como automática. Es importante fortalecer este proceso a través de capacitaciones a la población que va a utilizar la solución, así como el mejoramiento continuo de la gestión de los formularios.

\section{3 Resultados prueba 3}

En la Tabla III se presentan los registros de las mediciones reales, las mediciones obtenidas a través del software CROQUIS_UAV, y el porcentaje de error existente (para cada una de las tres medidas del experimento).

El error máximo obtenido, con un registro a 20 metros del suelo se encuentra cercano al $13 \%$. Los resultados obtenidos muestran una tendencia incremental en el porcentaje de error en la medida que el dron se aleja del piso. Esto se puede asociar a la baja resolución de la cámara utilizad (480 p) que obliga que la precisión (relación pixel/metro) se vea afectada al aumentar la distancia del registro.

\section{CONCLUSIONES}

El uso de herramientas tecnológicas (hardware, software y telecomunicaciones) para el mejoramiento del registro de accidentes de tránsito y su impacto en el tráfico y la salud pública se encuentra ampliamente documentada en el estado

TABLA III

ERRORES CON REGISTRO A 5 METROS

\begin{tabular}{|c|c|c|c|c|c|c|c|c|c|}
\hline ID_Med & $\begin{array}{c}\text { Medida } \\
\text { real }(\mathrm{m})\end{array}$ & $\begin{array}{c}\text { Medida } \\
\text { soft. altu- } \\
\text { ra 5 me- } \\
\text { tros }(\mathrm{m})\end{array}$ & $\begin{array}{c}\text { Error regis- } \\
\text { tro altura 5 } \\
\text { metros }(\%)\end{array}$ & $\begin{array}{c}\text { Medida } \\
\text { soft. altu- } \\
\text { ra 10 me- } \\
\text { tros }(\mathrm{m})\end{array}$ & $\begin{array}{c}\text { Error registro } \\
\text { altura 10 } \\
\text { metros }(\%)\end{array}$ & $\begin{array}{c}\text { Medida } \\
\text { soft altu- } \\
\text { ra 15 me- } \\
\text { tros }(\mathrm{m})\end{array}$ & $\begin{array}{c}\text { Error registro } \\
\text { altura 15 } \\
\text { metros }(\%)\end{array}$ & $\begin{array}{c}\text { Medida } \\
\text { soft. altu- } \\
\text { ra 20 me- } \\
\text { tros }(\mathrm{m})\end{array}$ & $\begin{array}{c}\text { Error regis- } \\
\text { tro altura 20 } \\
\text { metros }(\%)\end{array}$ \\
\hline 1 & 1 & 1 & 0,0 & 0,95 & 5,0 & 0,88 & 12,0 & 1,13 & 13,0 \\
\hline 2 & 3,5 & 3,3 & 5,7 & 3,3 & 5,7 & 3,2 & 8,6 & 3,14 & 10,3 \\
\hline 3 & 1,4 & 1,38 & 1,4 & 1,3 & 7,1 & 1,26 & 10,0 & 1,26 & 10,0 \\
\hline
\end{tabular}

Fuente: Los autores. 
de arte. El Gobierno colombiano, a través de la Resolución 11268 del 2012 del Ministerio de Transporte, ha delegado en cada ente responsable del transporte en los municipios y departamentos la tarea de implementar las soluciones que faciliten el seguimiento al fenómeno de la accidentalidad, a través del registro eficiente del Informe Policial de Accidentes de Tránsito y el Registro Nacional de Accidentes de Tránsito. En la experiencia compartida en el presente artículo se propone una solución que integra dispositivos de registro (terminales) digitales, software para la captación y almacenamiento de la información del IPAT, su transferencia a través de plataforma tecnológica en línea y su posterior visualización. Esta solución integra también una estrategia novedosa para la creación del Croquis del accidente como un elemento clave en el proceso probatorio.

Se concluye que el soporte tecnológico puede contribuir a mejorar los tiempos de registro del IPAT, siempre y cuando se garantice la correcta capacitación del personal involucrado. A su vez, se ha observado que este tiempo se ve incrementado en relación con la edad de los usuarios, probablemente asociado a las competencias digitales de estos. Otra ventaja de utilizar este tipo de tecnologías es que se disminuye la posibilidad de tener errores humanos en los procesos de registro y transcripción manual de la información, pues los datos digitados en la plataforma son transmitidos de forma confiable a la base de datos alojada en el servidor en línea.

Para futuros trabajos es necesario seguir explorando estrategias para facilitar el registro de la información, así como la entrega de soportes a los involucrados (impresión). Es fundamental también la producción de una versión del aplicativo "llave en mano" para ser implementado por los responsables del tema tránsito y transporte en el país. Proponer otras estrategias para registrar el croquis es necesario.

Finalmente, el uso de los UAV para tomar imágenes desde una vista superior del accidente permite contar con detalles y elementos probatorios que faciliten los procesos. Si bien puede observarse que cada equipo de levantamiento de accidentes cuente con un UAV para la toma de estas imágenes, la reducción de costos de estos y la calidad de los registros puede servir para ser aplicado en casos especiales.

\section{REFERENCIAS}

[1] OICA, "Motorization rate 2015- WorldWide," World Vehicles in use - By country an typoe 2005-2015, 2016. [Online]. Available: http://www.oica.net/category/vehicles-in-use/.

[2] Instituto Nacional de Medicina Legal y Ciencias Forenses, "2017- Forensis - Datos para la vida," Bogotá, 2018.

[3] Congreso de la República de Colombia, Ley 769 de 2002. Colombia, pp. 1-123, 2002.

[4] Congreso de la República de Colombia, Ley 1383 de 2010. Colombia, 2010.

[5] Ministerio de Transporte, Colombia, Resolución 11268 de 06 de diciembre de 2012. Colombia, pp. 1-5, 2012.

[6] Ministerio de Transporte, Colombia, Resolución No 0011268 de 2012. Colombia, p. 98, 2012.

[7] World Health Organization, "Preventing injuries and violence: A guide for ministries of health," Washinton, 2007.

[8] K. Smith and B. Smith, "Forecasting the Clearance Time of Freeway Accidents," Virginia, 2001.

[9] Y. Liu, J. Maojing, and W. Yunpeng, "Traffic impact analysis of highway accident based on the shockwave theory," cnki, 2012.

[10] Y. He, Z. Liu, and S. Du, "Prediction of Recovery Time of Urban Traffic Accident Based on Active Flow-Split," in 2016 International Conference on Smart City and Systems Engineering (ICSCSE), pp. 7-9, 2016.

[11] H. A. Najada and I. Mahgoub, "Anticipation and alert system of congestion and accidents in VANET using Big Data analysis for Intelligent Transportation Systems," in 2016 IEEE Symposium Series on Computational Intelligence (SSCI), pp. 1-8, 2016.

[12] J. Zhang, Y. Yu, and Y. Lei, "The study on an optimized model of traffic congestion problem caused by traffic accidents," in 2016 Chinese Control and Decision Conference (CCDC), pp. 688-692, 2016.

[13] S. Kodagoda, A. Alempijevic, S. Sehestedt, and G. Dissanayake, "Towards improving driver situation awareness at intersections," in 2007 IEEE/RSJ International Conference on Intelligent Robots and Systems, pp. 3739-3744, 2007.

[14] Diricom, "Diseño Inteligente de Redes Vehiculares (VANETs),” 2014. [Online]. Available: http://diricom.lcc. uma.es/diricom/diricom-es/VANETs-es.html.

[15] S. M. Bilal, C. J. Bernardos, and C. Guerrero, "Position-based routing in vehicular networks: A survey," J. Netw. Comput. Appl., vol. 36, no. 2, pp. 685-697, 2013.

[16] P. S. Barani and N. E. Elizabeth, "Registration and verification of vehicles in VANET's," in 2015 International Conference on Communications and Signal Processing (ICCSP), pp. 1087-1092, 2015.

[17] A. Rasheed and S. Ajmal, "Traffic information, management amp;amp; emergency response service (TIMERs)," in 2014 National Software Engineering Conference, pp. 19-24, 2014.

[18] J. Goswami, S. Ghosh, S. Katiyar, and A. Majumder, "Development of a prototype to detect speed limit violation for better traffic management," in 2015 Eighth International Conference on Contemporary Computing (IC3), pp. 449-454, 2015. 
[19] H. Al Najada and I. Mahgoub, "Big vehicular traffic Data mining: Towards accident and congestion prevention," in 2016 International Wireless Communications and Mobile Computing Conference (IWCMC), 2016, pp. 256-261.

[20] M. Bouabdellah, F. E. Bouanani, and H. Ben-azza, "A secure cooperative transmission model in VANET using attribute based encryption," in 2016 International Conference on Advanced Communication Systems and Information Security (ACOSIS), 2016, pp. 1-6.

[21] M. Garai and N. Boudriga, "A secure QoS aware VANET," in 2016 International Conference on Advanced Communication Systems and Information Security (ACOSIS), 2016, pp. 1-6.

[22] Y.-K. Ki, J.-W. Kim, and D.-K. Baik, "A Traffic Accident Detection Model using Metadata Registry," in Fourth International Conference on Software Engineering Research, Management and Applications (SERA'06), 2006, pp. 255-259.

[23] D. Jiang and L. Delgrossi, "IEEE 802.11p: Towards an International Standard for Wireless Access in Vehicular Environments," in VTC Spring 2008 - IEEE Vehicular Technology Conference, 2008, pp. 2036-2040.

[24] C. Liu, G. Wang, W. Ning, X. Lin, L. Li, and Z. Liu, "Anomaly detection in surveillance video using motion direction statistics," in 2010 IEEE International Conference on Image Processing, 2010, pp. 717-720.

[25] S. Xia, J. Xiong, Y. Liu, and G. Li, "Vision-based traffic accident detection using matrix approximation," in 2015 10th Asian Control Conference (ASCC), 2015, pp. $1-5$.

[26] M. H. Eiza, Q. Ni, and Q. Shi, "Secure and Privacy-Aware Cloud-Assisted Video Reporting Service in 5G-Enabled Vehicular Networks," IEEE Trans. Veh. Technol., vol. 65, no. 10, pp. 7868-7881, Oct. 2016.

[27] P. T. A. Quang, K. Piamrat, K. D. Singh, and C. Viho, "Video Streaming Over Ad Hoc Networks: A QoE-Based Optimal Routing Solution," IEEE Trans. Veh. Technol., vol. 66, no. 2, pp. 1533-1546, Feb. 2017.
[28] I. xu, N. Arumugam, and y. Yang, "Video Packet Scheduling with Stochastic QoS for Cognitive Heterogeneous Networks," IEEE Trans. Veh. Technol., vol. pp, no. 99, p. 1, 2017.

[29] M. Karim, S. Sorour, and P. Sadeghi, “Network Coding for Video Distortion Reduction in Device-to-Device Communications," IEEE Trans. Veh. Technol., vol. pp, no. 99, p. 1, 2016.

[30] N. Vladimirovich and M. Aleksee, "Situation Control of Unmanned Aerial Vehicles for Road Traffic MOnitoring," Mod. Appl. Sci., vol. 9, no. 5, 2015.

[31] P. Fang, J. Lu, Y. Tian, and Z. Miao, "An Improved Object Tracking Method in UAV Videos," Procedia Eng., vol. 15, pp. 634-638, 2011.

[32] K. Bethke, S. Baumgartner, and M. Gabele, "Airborne Road Traffic Monitoring with Radar," 2007.

[33] K. Kanistras, G. Martins, M. J. Rutherford, and K. P. Valavanis, "Survey of Unmanned Aerial Vehicles (UAVs) for Traffic Monitoring," in Handbook of Unmanned Aerial Vehicles, K. P. Valavanis and G. J. Vachtsevanos, Eds. Dordrecht: Springer Netherlands, 2015, pp. 2643-2666.

[34] J. C. Martínez, "Manual diligenciamiento del IPAT," Bogotá, 2014.

[35] A. Puri, "A survey of unmanned aerial vehicles (UAV) for traffic surveillance," 2005.

[36] UNITY, "Flujo de trabajo de los Assets (Asset Workflow)," 2014. [Online]. Available: https://docs.unity3d. com/es/current/Manual/AssetWorkflow.html. [Accessed: 12-Jun-2018].

[37] Parrot, “Ar. Drone 2.0," 2014. [Online]. Available: https://www.parrot.com/es/drones/parrot-ardrone-20-elite-edition\#parrotardrone-20-elite-edition. [Accessed: 15-Jun-2018]. 